\title{
Hyperglycemia has no effect on development of restenosis after percutaneous transluminal angioplasty (PTA) in a diabetic rabbit model
}

\author{
Xiaojun Zhou, Jianjun Dong', Li Zhang ${ }^{2}$, Ju Liu ${ }^{3}$, Xiaofeng Dong ${ }^{4}$, Qing Yang, \\ Fupeng Liu and Lin Liao \\ Department of Endocrinology, Shandong Provincial Qianfoshan Hospital, Shandong University, \\ No. 16766, Jingshi Road, Lixia District, Jinan, Shandong Province, China \\ ${ }^{1}$ Department of Endocrinology, Qilu Hospital of Shandong University, Jinan, Shandong, China \\ ${ }^{2}$ Department of Sonography, and ${ }^{3}$ Laboratory of Microvascular Medicine, Medical Research Center, \\ Shandong Provincial Qianfoshan Hospital, Shandong University, Jinan, Shandong, China \\ ${ }^{4}$ Department of Hepatobiliary Surgery, the People's Hospital of Guangxi Zhuang Autonomous Region, \\ Nanning, Guangxi, China
}

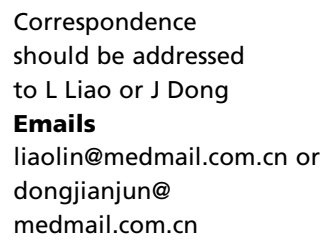

\begin{abstract}
It is well known that hyperglycemia is a trigger of atherosclerosis in patients with diabetes mellitus. However, the role of hyperglycemia in restenosis remains unclear. In this study, we investigated the effects of hyperglycemia on restenosis. Stenosis was evaluated in two sets of diabetic rabbit models: i) diabetic restenosis versus nondiabetic restenosis and ii) diabetic atherosclerosis versus nondiabetic atherosclerosis. Our results indicated that there was no difference in rates of stenosis between the diabetic and the nondiabetic groups in restenosis rabbit models. However, the incidence of stenosis was significantly higher in the diabetic atherosclerosis group compared with the nondiabetic atherosclerosis group. Similarly, the intima-media thickness and cell proliferation rate were significantly increased in the diabetic atherosclerosis group compared with the nondiabetic atherosclerosis group, but there was no difference between the diabetic restenosis and the nondiabetic restenosis groups. Our results indicate that hyperglycemia is an independent risk factor for atherosclerosis, but it has no evident effect on restenosis. These findings indicate that the processes of atherosclerosis and restenosis may involve different pathological mechanisms.
\end{abstract}

\section{Key Words}

- hyperglycemia

- restenosis

- percutaneous transluminal angioplasty

- atherosclerosis

\section{Introduction}

Peripheral vascular disease (PVD), one of the major clinical manifestations of atherosclerosis, is highly prevalent in diabetic patients, and may cause intermittent claudication and critical limb ischemia (Bosevski 2012, Delbin \& Trask 2014). Revascularization is often recommended for patients who are resistant to conservative therapies.
Although surgical bypass grafting is the gold standard, acceptable midterm secondary patency and limb salvage rates have led us to consider percutaneous transluminal angioplasty (PTA) as the first-line treatment for patients with lower extremity ischemia (Abularrage et al. 2010). Successful revascularization of the lower limbs by PTA

Published by Bioscientifica Ltd. 
can improve patients' quality of life and functional status and decrease future atherothrombotic events (Giugliano et al. 2013). However, the application of PTA to PVD is largely limited due to the high restenosis rate (Jones et al. 2013).

Intimal hyperplasia is a major process of restenosis after PTA. In the diabetic state, hyperglycemia, which can enhance intimal hyperplasia by induction of platelet hypersensitivity, dyslipidemia, and dysregulation of expression of chemotactic factors (Barbieri et al. 2013, Zeadin et al. 2013), is potentially related to the process of restenosis. However, conflicting results have been reported with respect to the role of hyperglycemia in the pathogenesis of restenosis (Aronson et al. 1996, Park et al. 2001, Mazeika et al. 2003, Carter 2004, Lindsay et al. 2007, Lavi et al. 2008, Saxon et al. 2008).

To assess the effects of hyperglycemia on restenosis after PTA, we developed models of restenosis in New Zealand white rabbits (Chatterjee et al. 2014). Diabetes was induced by injection of alloxan. Stenosis rate, intimamedia thickness, cell proliferation rate, and their relationship with glucose levels were analyzed. Rabbit models of atherosclerosis were also established. The effects of hyperglycemia on models of restenosis and atherosclerosis were studied.

\section{Materials and methods}

\section{Animals and treatments}

Three-to-four-months-old male New Zealand white rabbits were obtained from the Animal Center of Shandong Agriculture Science Academy, China. All rabbits were allowed free access to food and water during the whole study. The rabbits were allowed to acclimatize for at least 7 days, and then were fed with a high-cholesterol diet (1\% cholesterol) thereafter. All animal care and experimental procedures were in accordance with the guide for the care and use of laboratory animals published by the Chinese National Institutes of Health, and the protocol was approved by the ethnical committee of Shandong University Medical School. All surgical procedures were performed under anesthesia with sodium pentobarbital.

All rabbits were randomly divided into four groups: the diabetic restenosis group, nondiabetic restenosis group, diabetic atherosclerosis group, and nondiabetic atherosclerosis group. After 1 week of a high-cholesterol diet, 24 rabbits received injections of alloxan $(80 \mathrm{mg} / \mathrm{kg})$ via their ear veins to induce type 1 diabetes. As blood glucose levels should become stable within a week or so
(Hadcock et al. 1991, Hao et al. 2013), blood glucose concentrations were measured 1 week after injection of alloxan. Sixteen rabbits with fasting plasma glucose levels of over $300 \mathrm{mg} / \mathrm{dl}$ were considered to be diabetic, and divided into two groups: the diabetic atherosclerosis group $(n=8)$ and the diabetic restenosis group $(n=8)$. Another 16 agematched rabbits which received injections of saline into their ear veins instead of alloxan were used as nondiabetic controls, and assigned to the nondiabetic atherosclerosis group $(n=8)$ and the nondiabetic restenosis group $(n=8)$. No exogenous insulin and hypoglycemic agents were given to these animals during the whole study period.

\section{Surgical procedures for production of models of restenosis and atherosclerosis}

One week after the injection of alloxan $(80 \mathrm{mg} / \mathrm{kg})$ or saline mentioned above, we started to develop models of restenosis and atherosclerosis in both diabetic and nondiabetic rabbits.

Restenosis models Rabbits were anesthetized with phenobarbital. An arteriotomy in the right saphenous artery was made with standard surgical techniques (Fig. 1A). A $2.5-\mathrm{mm}$, wire-guided balloon catheter was inserted into the iliac artery (the length was about $15 \mathrm{~cm}$ ) through the incision (Fig. 1B). The injury consisted of three gentle tractions of the balloon catheter at 8,10 , and $12 \mathrm{~atm}$ to ensure complete endothelial denudation. At the end of the procedure, the balloon catheter was withdrawn, the vessel was ligated, and the surgical site was closed by a simple continuous intradermal pattern with a 4-0 polydioxanone suture. After surgery, the rabbits received i.m. injections of 800000 units of penicillin daily for 3 days. In order to induce restenotic plaque, PTA was performed if the iliac artery developed severe atherosclerotic damage (about 4 weeks after the first surgical procedure), which was confirmed by ultrasound examination (Visualsonics, Toronto, ON, Canada) (Fig. 1E). After anesthesia, a midsagittal incision was made in the dissected distal ends of the right femoral artery (Fig. 1C). A 2.5-mm, wire-guided balloon catheter was inserted into the narrow iliac artery and the balloon was inflated to $12 \mathrm{~atm}$ three times for $5 \mathrm{~s}$ each time at the site of stenosis (Fig. 1D). After the dilatation, we removed the catheter and ligated the femoral artery. The rabbits received an i.m. injection of penicillin at the dose of 800000 units daily for 3 days. Another color Doppler ultrasonogram was performed to demonstrate the patency of the stenosed iliac arteries after PTA (Fig. 1F).

Published by Bioscientifica Ltd. 
Balloon-induced endothelial injury

A

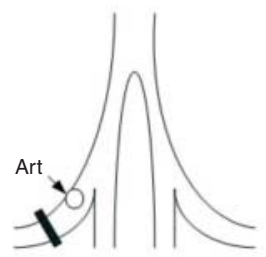

PTA

C

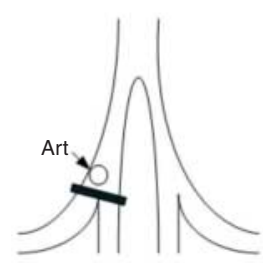

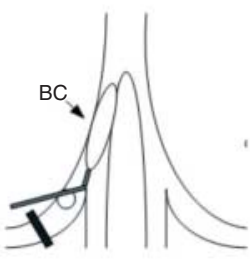

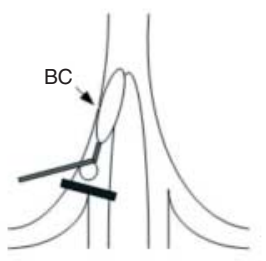

E

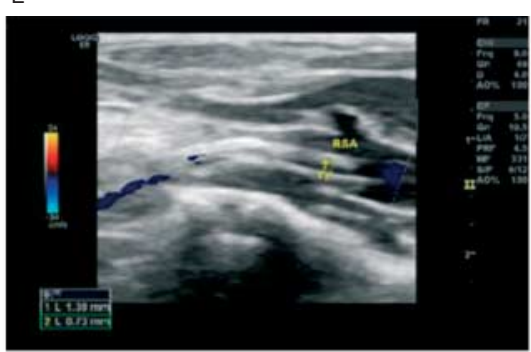

G

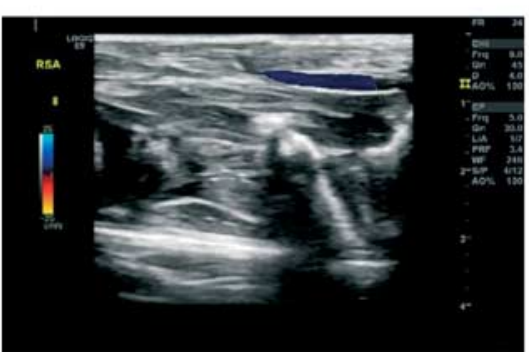

$\mathrm{F}$

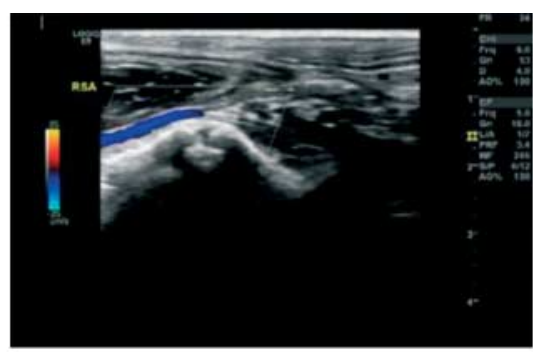

$\mathrm{H}$

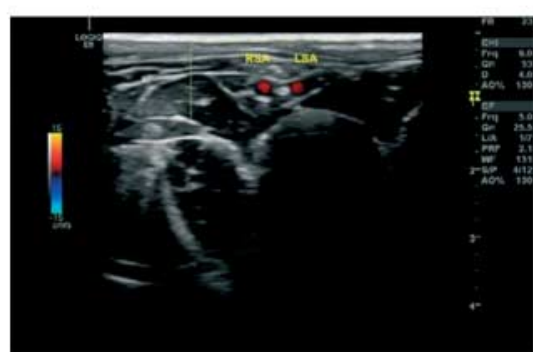

\section{Figure 1}

(A) After isolated, the distal saphenous artery was ligated. A small arteriotomy (Art) was made in the proximal saphenous artery. (B) A balloon catheter (BC) was advanced into the femoral artery and iliac artery (after topical application of lidocaine). Then three gentle tractions of the $B C$ at 8,10 , and 12 atm were made to ensure complete endothelial denudation. (C) Four weeks after the first surgery, a midsagittal incision was made in the dissected distal ends of the femoral artery. (D) A BC was inserted into the narrowed iliac artery and

Atherosclerosis models On the same day, as the rabbits in restenosis groups underwent PTA surgery, ballooninduced injuries of the right iliac arteries (with no plaques, Fig. $1 \mathrm{G}$ and $\mathrm{H}$ ) were performed on rabbits in nondiabetic and diabetic atherosclerosis groups to develop models of atherosclerosis.

\section{Tissue harvesting and histological processing}

For assessment of neointimal hyperplasia, the animals were killed under anesthesia on day 28 after the last surgical procedure. Blood samples were collected from the ear veins and HbA1c levels were evaluated using a commercial diagnostic kit (Nanjing Jiancheng Bioengineering Institute, Nanjing, China). The injured iliac arteries were cut into $3 \mathrm{~mm}$ pieces. Five segments were fixed in $10 \%$ neutral buffered formalin solution, embedded in paraffin, and processed for histopathological analysis. The sections $(5 \mu \mathrm{m})$ were stained with hematoxylin and eosin (HE) for general appearance, Masson's trichrome for collagen, and elastic van-Gieson dye for elastin and observed by microscopy.

Histomorphometric analysis was performed as described previously (Ali et al. 2007). Total vessel area inflated at 12 atm three times for 5 s each at the site of stenosis. (E) Four weeks after Balloon-induced endothelial injuries, severe iliac artery stenosis were formed in models of restenosis. (F) Results of color Doppler ultrasonography demonstrated the patency of the stenosed iliac arteries after PTA in models of restenosis. ( $\mathrm{G}$ and $\mathrm{H}$ ) Results of color Doppler ultrasonography demonstrated blood flow through normal arteries in models of atherosclerosis when severe iliac artery stenosis were formed in models of restenosis.

and the area of lumen, media, and neointima were measured using Image Pro-Plus Software (Image Pro-Plus 6.0, Media Cybernetics, Silver Spring, MD, USA).

Intimal growth was estimated from the ratio of the intimal area to the area bounded by the internal elastic lamina (luminal cross-sectional area narrowing) and the ratio of intimal to medial areas.

\section{Immunohistochemical analysis}

Tissue sections $(4 \mu \mathrm{m})$ deparaffinized in xylene and rehydrated through graded alcohol washes were incubated with $1 \% \mathrm{H}_{2} \mathrm{O}_{2}$ in methanol for $10 \mathrm{~min}$ to block endogenous peroxidase activity. The sections were then incubated overnight in a humid chamber at $4^{\circ} \mathrm{C}$ with primary antibody against: proliferating cell nuclear antigen (PCNA; Merck, Millipore, Darmstadt, Germany) and $\alpha$-smooth muscle actin (Abcam, Inc., Cambridge, MA, USA). After washing with $1 \times$ PBS, sections were incubated with biotinylated antimouse secondary antibody (Zhongshan, Beijing, China) for 30 min. Diaminobenzidinetetrahydrochloride was used to visualize the staining. The samples were counterstained with hematoxylin before addition of coverslips. The primary antibodies were omitted for negative control sections.

Published by Bioscientifica Ltd 
PCNA-positi ve cells were counted in ten randomly selected $200 \times$ high-power fields under a microscope. The PCNA index was calculated according to the following formula: number of PCNA-positive cells/total cell count $\times 100 \%$.

\section{Statistical analyses}

All data are presented as mean \pm s.D. Comparisons were made using one-way ANOVA with SPSS Software (version 20.0, SPSS China). A value of $P<0.05$ was considered statistically significant.

\section{Results}

\section{Animals}

The experiment was carried out using 40 New Zealand white rabbits. Baseline nonfasting blood glucose levels were normal in all animals (Table 1). Of the 24 alloxantreated rabbits, 16 developed hyperglycemia; eight were excluded due to inadequate glucose levels. After all surgical procedures, three animals (two in the diabetic atherosclerosis group and one in the diabetic restenosis group) died due to severe hyperglycemia. Three rabbits (two in the nondiabetic atherosclerosis group and one in the nondiabetic restenosis group) died during ultrasound examination and three (one in the nondiabetic restenosis group and two in the diabetic atherosclerosis group) died of thrombosis. Before harvesting of tissue, six rabbits remained in the nondiabetic atherosclerosis group, four in the diabetic atherosclerosis group, six in the nondiabetic restenosis group, and seven in the diabetic restenosis group. Rabbits in the diabetic groups had a slight weight gain, while the levels of HbA1c were notably higher than those of rabbits in the nondiabetic groups (Table 1). The blood glucose levels of rabbits in each group are given in Table 1.

\section{Effects of hyperglycemia on rate of stenosis and intima/ media areas of the atherosclerotic and restenotic plaques}

The samples were collected from the iliac arteries of rabbits undergoing different treatments. A progressive increase in intimal cross-sectional area of the iliac arteries was detected in all four groups (Fig. 2A).

The rate of stenosis in the diabetic restenosis group $(92.33 \pm 5.46 \%)$ was not significantly different from the nondiabetic restenosis $(89.17 \pm 7.14 \%)$ group $(P>0.05)$. Whereas the diabetic atherosclerosis group showed a higher stenosis rate $(72.37 \pm 24.55 \%)$ than the nondiabetic atherosclerosis group $(35.54 \pm 40.97 \%)(P<0.05)$ (Fig. $2 \mathrm{~B})$. The calculated intima/media ratio results were consistent with stenosis rates (Fig. 2C). The intima/media ratio in the diabetic restenosis group was $2.39 \pm 0.43$ compared with $2.13 \pm 0.88$ in the nondiabetic restenosis group $(P>0.05)$, and $1.55 \pm 0.47$ in the diabetic atherosclerosis group compared with $0.37 \pm 0.43$ in the nondiabetic atherosclerosis group $(P<0.05)$.

\section{Effects of hyperglycemia on cell proliferation in the atherosclerotic and restenotic plaques}

A large number of PCNA-positive cells were detected in the intima and media, and the cell arrangement was extremely disordered. The PCNA index in the diabetic atherosclerosis group was significantly higher than that in the nondiabetic atherosclerosis group $(P<0.05)$. In contrast, in both the diabetic and nondiabetic restenosis groups, cell proliferation was both markedly increased in the restenotic plaques and no significant difference was found between these two groups (Fig. 3).

\section{Smooth muscle cells content changes}

As revealed by immunohistochemical staining for $\alpha$-smooth muscle actin (a marker for smooth muscle cells (SMCs)), the number of SMCs was higher in restenotic plaques than in

Table 1 Characteristics of animals. Blood glucose levels were measured 1 week after injection of alloxan

\begin{tabular}{|c|c|c|c|c|}
\hline \multicolumn{5}{|l|}{ Weight (g) } \\
\hline Initial & $2160.4 \pm 216.6$ & $2061.1 \pm 127.7$ & $2173.3 \pm 178.5$ & $2028.3 \pm 173.7$ \\
\hline At death & $3143.0 \pm 426.4$ & $2217.5 \pm 291.7$ & $2976.7 \pm 307.0$ & $2096.6 \pm 320.0$ \\
\hline Glucose (mg/dl) & $104.76 \pm 12.24$ & $366.84 \pm 70.38 *$ & $101.16 \pm 11.70$ & $391.50 \pm 90.54 *$ \\
\hline
\end{tabular}

${ }^{*} P<0.05$ versus control group in each diabetic model. NDA, nondiabetic atherosclerosis rabbits; DA, diabetic atherosclerosis rabbits; NDR, nondiabetic restenosis rabbits; DR, diabetic restenosis rabbits; HbA1c, glycosylated hemoglobin; ABS, absorbance.

http://joe.endocrinology-journals.org DOI: $10.1530 / J O E-14-0391$
() 2015 Society for Endocrinology Printed in Great Britain
Published by Bioscientifica Ltd 
A
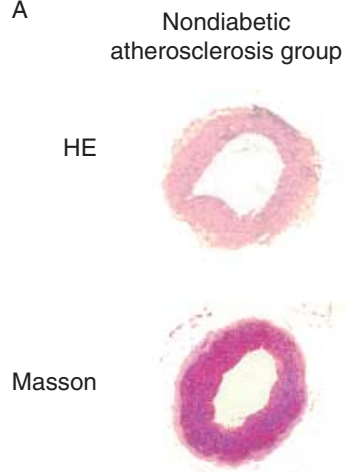

VGF

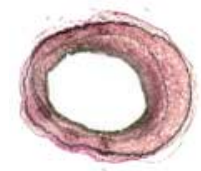

Diabetic atherosclerosis group
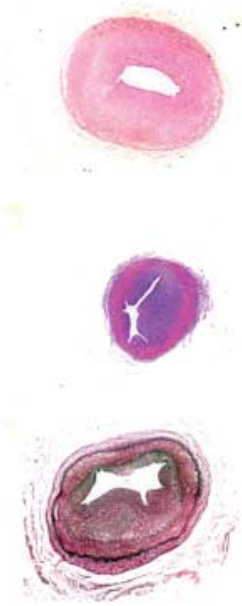

Nondiabetic restenosis group
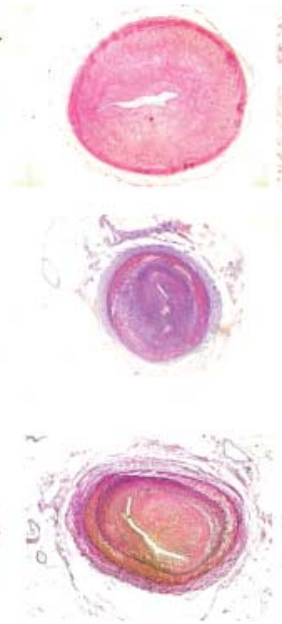

Diabetic

restenosis group
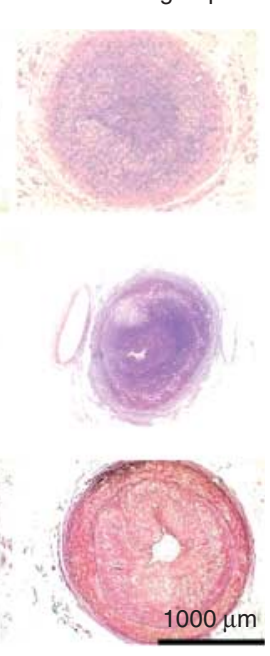

B

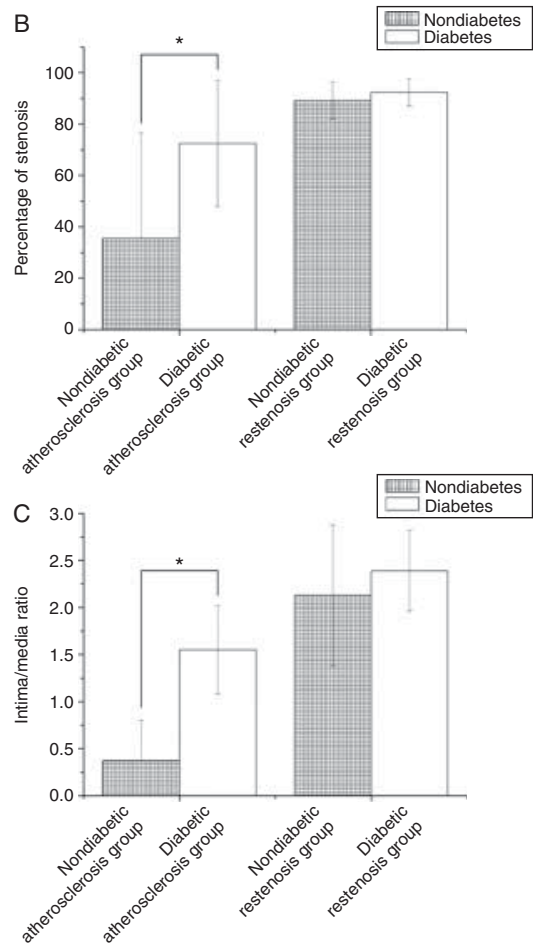

\section{Figure 2}

(A) Cross sections stained with HE, Masson, and VGF. (B) Rates of stenosis of the right iliac arteries of rabbits in four groups. (C) Intima:media ratio of the right iliac arteries of rabbits in four groups. Magnification $40 \times$.

atherosclerotic plaques. In samples from both diabetic and nondiabetic models, large number of SMCs were observed in the intima of restenotic plaques, whereas fewer SMCs were found in the atherosclerotic plaques (Fig. 4).

\section{Discussion}

Restenosis after PTA in diabetic patients with PVD is an unsolved clinical issue. Factors contributing to restenosis remain poorly defined. In this study, we investigated the effects of hyperglycemia on restenosis in two sets of diabetic rabbit models: restenosis and atherosclerosis models. We found that i) build-up of atherosclerotic plaques was much faster in diabetic rabbits than in nondiabetic rabbits; ii) formation of restenotic plaques in diabetic restenosis rabbits was not different from that in nondiabetic restenosis rabbits and iii) numbers of PCNA-positive cells in diabetic atherosclerotic plaques were higher than those in nondiabetic atherosclerotic plaques; whereas in diabetic restenotic and nondiabetic restenotic plaques, numbers of PCNA-positive cells were not significantly different. These findings indicate that hyperglycemia is a risk factor for atherosclerosis but there was no evident effect on the process of restenosis.
*Statistically significant difference $(P<0.05)$ from the respective control. Scale bar $=1000 \mu \mathrm{m}$.

Hyperglycemia, a hallmark of diabetes, has been well accepted as a major risk factor for atherosclerosis (Barbieri et al. 2013, Nagareddy et al. 2013). In addition, it might potentiate the response to arterial injury and be involved in multiple steps in the process of restenosis. In this study, we confirmed that hyperglycemia is closely correlated with atherosclerosis; however, it had no obvious effect on restenosis.

Restenosis is a secondary injury to atherosclerotic vessels. Restenotic lesions cause structural changes in the arteries. The mechanisms underlying the formation of restenotic plaques may be different from those for the primary atherosclerotic plaques. Atherosclerosis is an inflammatory process, during which endothelial cells, monocytes, and $\mathrm{T}$ lymphocytes interact with each other. Hyperglycemia can accelerate inflammatory processes via the activation of multiple signaling pathways, and finally leads to the development of atherosclerosis. Whereas inflammation does not appear to be essential in the development of restenosis, results from previous studies indicated that there are almost no white blood cells (WBCs) in restenotic plaques (Roque et al. 2002, Edlin et al. 2009). Therefore, hyperglycemia, which accelerates the process of atherosclerosis mainly by promoting

Published by Bioscientifica Ltd 
Nondiabetic atherosclerosis group

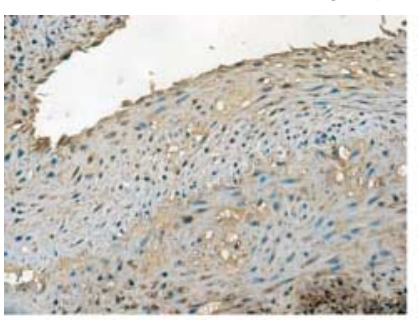

Nondiabetic restenosis group

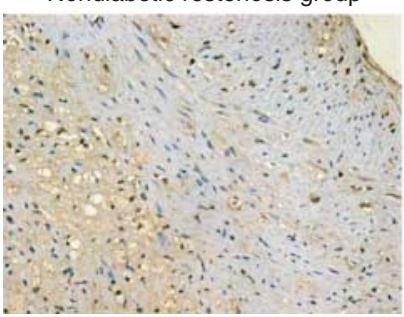

Diabetic atherosclerosis group

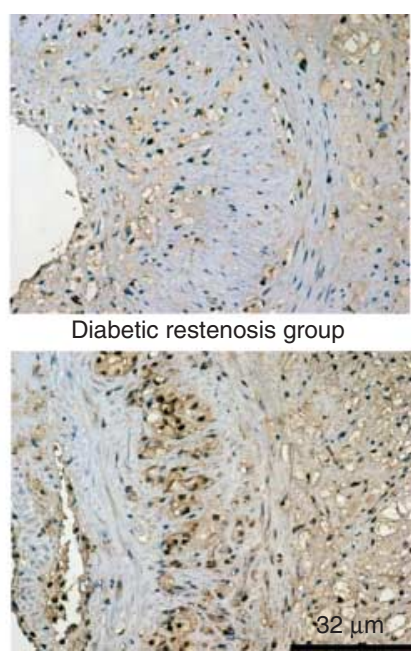

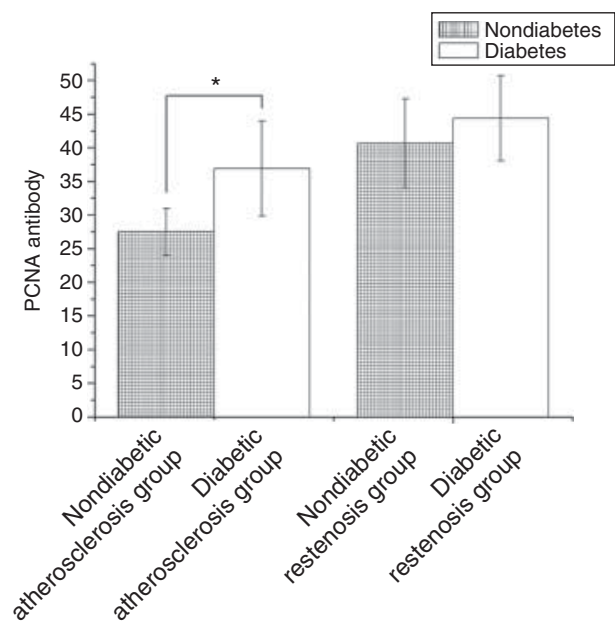

Figure 3

Immunohistochemistry of atherosclerotic and restenotic plaques in diabetic and nondiabetic rabbits. Representative sections are shown. Staining for PCNA. Magnification $200 \times$. Quantification of positive

inflammatory responses, may have little effect on the development of restenosis.

So what accounts for rapid formation of restenotic plaques? Unlike atherosclerotic plaques in which WBCs and inflammation are major mediators (Roque et al. 2002, Edlin et al. 2009, Barbieri et al. 2013, Nagareddy et al. 2013), restenotic plaques is typically hypercellular with foci of vascular SMCs and extracellular matrix (ECM) (Zargham 2008, Muthiah et al. 2014) (Fig. 4). In the acute phase of restenotic plaques, arterial injury evokes loss of the contractile phenotype in the tunica media, leading to migration of SMCs from the media toward the intima. Then the presence of these SMCs result in the intimal thickening of the restenosis via the excessive synthesis of ECM and cell proliferation (Zargham 2008). Thus, the migration of vascular SMCs from the media to the intima may be the principal step in the development of restenosis (Marx et al. 2011), and restenotic lesion-activated specific signaling pathways leading to the migration of SMCs may play essential roles in this process.

In addition, we found a strong trend toward a higher stenosis rate and cell proliferation activity in the nondiabetic restenosis group compared with the diabetic atherosclerosis group, indicating that migration of SMCs to arterial plaques may play a key role in the pathogenesis of restenosis.

In this study, we used alloxan to establish models of type 1 diabetes and observed only the effects of hyperglycemia on restenosis. The influence of hyperinsulinemia immunostaining in four groups expressed as a ratio of positive:total cells \pm error. *, Statistically significant difference $(P<0.05)$ between the indicated groups. Scale bar $=32 \mu \mathrm{m}$.

and insulin resistance on restenosis was not investigated. Insulin has several biological activities, which may be related to the process of restenosis. Therefore, future studies are needed with models of type 2 diabetes. In addition, due to the complex surgeries and the long observation time, there were only four animals alive in the diabetic atherosclerosis group, which may weaken the statistical power to some degree.
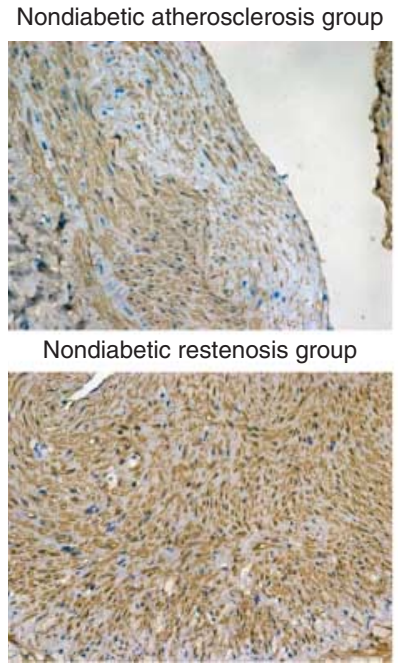
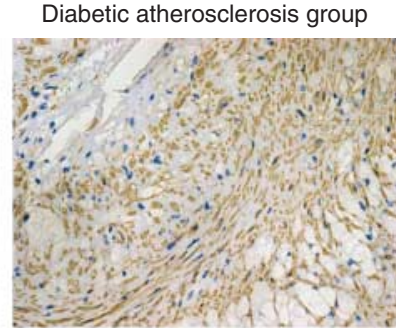

Diabetic restenosis group

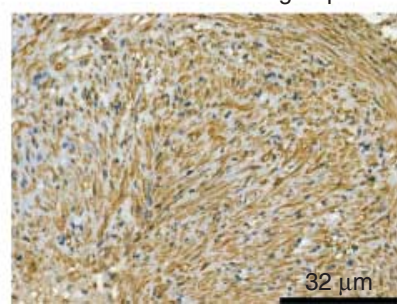

\section{Figure 4}

Immunohistochemistry of atherosclerotic and restenotic plaques in diabetic and nondiabetic rabbits. Representative sections are shown. Magnification $200 \times$. Scale bar $=32 \mu \mathrm{m}$. 
In summary, we demonstrated that hyperglycemia plays a critical role in the development of atherosclerosis, but has little effect on restenosis after PTA, indicative of distinct mechanisms for these processes. Further studies are needed to explore the mechanisms underlying the formation of restenosis in both animal models and human.

\section{Declaration of interest}

The authors declare that there is no conflict of interest that could be perceived as prejudicing the impartiality of the research reported.

\section{Funding}

This work was supported by grants from the National Natural Scientific Foundation of China (ZR2010HM044), Shandong Provincial Science \& Technology Development Program, China (2012GGH11862) and Chinese Medical Association Foundation (0910340189).

\section{References}

Abularrage CJ, Conrad MF, Hackney LA, Paruchuri V, Crawford RS, Kwolek CJ, LaMuraglia GM \& Cambria RP 2010 Long-term outcomes of diabetic patients undergoing endovascular infrainguinal interventions. Journal of Vascular Surgery 52 314-322 e311-e314. (doi:10.1016/j.jvs.2010.03.015)

Ali ZA, Alp NJ, Lupton H, Arnold N, Bannister T, Hu Y, Mussa S, Wheatcroft M, Greaves DR, Gunn J et al. 2007 Increased in-stent stenosis in ApoE knockout mice: insights from a novel mouse model of balloon angioplasty and stenting. Arteriosclerosis, Thrombosis, and Vascular Biology 27 833-840. (doi:10.1161/01.ATV.0000257135.39571.5b)

Aronson D, Bloomgarden Z \& Rayfield EJ 1996 Potential mechanisms promoting restenosis in diabetic patients. Journal of the American College of Cardiology 27 528-535. (doi:10.1016/0735-1097(95)00496-3)

Barbieri M, Rizzo MR, Marfella R, Boccardi V, Esposito A, Pansini A \& Paolisso G 2013 Decreased carotid atherosclerotic process by control of daily acute glucose fluctuations in diabetic patients treated by DPP-IV inhibitors. Atherosclerosis 227 349-354. (doi:10.1016/j.atherosclerosis.2012.12.018)

Bosevski M 2012 Peripheral arterial disease and diabetes. Prilozi 33 65-78.

Carter AJ 2004 TOR of the cell cycle: are there important implications for diabetics in the era of the drug-eluting stent? Catheterization and Cardiovascular Interventions 61 233-236. (doi:10.1002/ccd.10764)

Chatterjee S, Bedja D, Mishra S, Amuzie C, Avolio A, Kass D, Berkowitz D \& Renehan M 2014 Inhibition of glycosphingolipid synthesis ameliorates atherosclerosis and arterial stiffness in Apo $\mathrm{E}^{-1-}$ mice and rabbits fed a high fat and cholesterol diet. Circulation 129 2403-2413. (doi:10.1161/ CIRCULATIONAHA.113.007559)

Delbin MA \& Trask AJ 2014 The diabetic vasculature: physiological mechanisms of dysfunction and influence of aerobic exercise training in animal models. Life Sciences 102 1-9. (doi:10.1016/j.lfs.2014.02.021)

Edlin RS, Tsai S, Yamanouchi D, Wang C, Liu B \& Kent KC 2009 Characterization of primary and restenotic atherosclerotic plaque from the superficial femoral artery: potential role of Smad3 in regulation of SMC proliferation. Journal of Vascular Surgery 49 1289-1295. (doi:10.1016/j.jvs.2008.11.096)

Giugliano G, Di Serafino L, Perrino C, Schiano V, Laurenzano E, Cassese S, De Laurentis M, Schiattarella GG, Brevetti L, Sannino A etal. 2013 Effects of successful percutaneous lower extremity revascularization on cardiovascular outcome in patients with peripheral arterial disease. International Journal of Cardiology 167 2566-2571. (doi:10.1016/j.ijcard. 2012.06.055)

Hadcock S, Richardson M, Winocour PD \& Hatton MW 1991 Intimal alterations in rabbit aortas during the first 6 months of alloxan-induced diabetes. Arteriosclerosis and Thrombosis 11 517-529. (doi:10.1161/01. ATV.11.3.517)

Hao E, Lee SH \& Levine F 2013 Efficient $\beta$-cell regeneration by a combination of neogenesis and replication following $\beta$-cell ablation and reversal of pancreatic duct ligation. Stem Cells 31 2388-2395. (doi:10.1002/stem.1492)

Jones DW, Schanzer A, Zhao Y, MacKenzie TA, Nolan BW, Conte MS, Goodney PP \& Vascular Study Group of New England 2013 Growing impact of restenosis on the surgical treatment of peripheral arterial disease. Journal of the American Heart Association 2 e000345. (doi:10.1161/JAHA.113.000345)

Lavi S, Kapeliovich M, Gruberg L, Roguin A, Boulos M, Grenadier E, Amikam S, Markiewicz W, Beyar R \& Hammerman H 2008 Hyperglycemia during acute myocardial infarction in patients who are treated by primary percutaneous coronary intervention: impact on long-term prognosis. International Journal of Cardiology 123 117-122. (doi:10.1016/j.ijcard.2006.11.222)

Lindsay J, Sharma AK, Canos D, Nandalur M, Pinnow E, Apple S, Ruotolo G, Wijetunga M \& Waksman R 2007 Preprocedure hyperglycemia is more strongly associated with restenosis in diabetic patients after percutaneous coronary intervention than is hemoglobin A1C. Cardiovascular Revascularization Medicine 8 15-20. (doi:10.1016/j.carrev.2006.10.002)

Marx SO, Totary-Jain H \& Marks AR 2011 Vascular smooth muscle cell proliferation in restenosis. Circulation. Cardiovascular Interventions 4 104-111. (doi:10.1161/CIRCINTERVENTIONS.110.957332)

Mazeika P, Prasad N, Bui S \& Seidelin PH 2003 Predictors of angiographic restenosis after coronary intervention in patients with diabetes mellitus. American Heart Journal 145 1013-1021. (doi:10.1016/S00028703(03)00085-1)

Muthiah M, Islam MA, Cho CS, Hwang JE, Chung IJ \& Park IK 2014 Substrate-mediated delivery of microRNA-145 through a polysorbitolbased osmotically active transporter suppresses smooth muscle cell proliferation: implications for restenosis treatment. Journal of Biomedical Nanotechnology 10 571-579. (doi:10.1166/jbn.2014.1737)

Nagareddy PR, Murphy AJ, Stirzaker RA, Hu Y, Yu S, Miller RG, Ramkhelawon B, Distel E, Westerterp M, Huang LS et al. 2013 Hyperglycemia promotes myelopoiesis and impairs the resolution of atherosclerosis. Cell Metabolism 17 695-708. (doi:10.1016/j.cmet.2013.04.001)

Park SH, Marso SP, Zhou Z, Foroudi F, Topol EJ \& Lincoff AM 2001 Neointimal hyperplasia after arterial injury is increased in a rat model of non-insulin-dependent diabetes mellitus. Circulation 104 815-819. (doi:10.1161/hc3301.092789)

Roque M, Kim WJ, Gazdoin M, Malik A, Reis ED, Fallon JT, Badimon JJ, Charo IF \& Taubman MB 2002 CCR2 deficiency decreases intimal hyperplasia after arterial injury. Arteriosclerosis, Thrombosis, and Vascular Biology 22 554-559. (doi:10.1161/hq0402.105720)

Saxon RR, Dake MD, Volgelzang RL, Katzen BT \& Becker GJ 2008 Randomized, multicenter study comparing expanded polytetrafluoroethylene-covered endoprosthesis placement with percutaneous transluminal angioplasty in the treatment of superficial femoral artery occlusive disease. Journal of Vascular and Interventional Radiology 19 823-832. (doi:10.1016/j.jvir.2008.02.008)

Zargham R 2008 Preventing restenosis after angioplasty: a multistage approach. Clinical Science 114 257-264. (doi:10.1042/CS20070228)

Zeadin MG, Petlura CI \& Werstuck GH 2013 Molecular mechanisms linking diabetes to the accelerated development of atherosclerosis. Canadian Journal of Diabetes 37 345-350. (doi:10.1016/j.jcjd.2013.06.001)

Received in final form 1 November 2014

Accepted 10 November 2014

Accepted Preprint published online 10 November 2014

Published by Bioscientifica Ltd. http://joe.endocrinology-journals.org

DOI: 10.1530/JOE-14-0391
C) 2015 Society for Endocrinology Printed in Great Britain 